\title{
CAMBIOS EN LA CALIDAD DE LA SEMILLA DE MAÍZ DURANTE SU DESARROLLO Y MADURACIÓN1
}

\author{
Mariano Mendoza Elos ${ }^{2}$, Luis Latournerie ${ }^{3}$, Ernesto Moreno ${ }^{4}$, Guillermo Castañón ${ }^{5}$, José Cruz Carrillo 6 , \\ Carlos De León ${ }^{7}$, J. Guadalupe García ${ }^{2}$
}

\begin{abstract}
RESUMEN
Cambios en la calidad de la semilla de maíz durante su desarrollo y maduración. El objetivo del presente estudio fue investigar los cambios en el desarrollo y maduración de la semilla en la germinación, vigor de plántula, peso volumétrico y peso de 1.000 semillas. El trabajo experimental se realizó en Celaya, Guanajuato, México durante el 2001. La variedad sintética Roque 1 fue sembrada en dos repeticiones, en dos surcos de cinco metros de longitud, espaciados a $0,75 \mathrm{~m}$ y dos semillas por golpe. Los muestreos se empezaron a realizar 30 días después de la floración. Se cosecharon ocho mazorcas por tratamiento, las cuales fueron desgranados en forma manual. El diseño experimental fue completamente al azar con arreglo factorial. Las medias de la característica de germinación y vigor presentaron un mismo patrón de comportamiento como índices de maduración de la semilla. El peso volumétrico se incrementó a $71 \mathrm{~kg} / \mathrm{ha}$ a los 130 días y el peso de 1000 semillas no presentó un efecto significativo en la calidad fisiológica de la semilla. El máximo porcentaje de germinación y vigor se encontró cuando la humedad de la semilla se redujo de 40 a $20 \%$. La respuesta de los muestreos de 106 a 121 días después de la siembra fue pobre para determinar la calidad de la semilla. También se encontró mayor vigor y germinación en la semilla ubicada en la parte basal y media de la mazorca. Estos resultados revelan que la madurez fisiológica no es el mejor indicador de la máxima calidad de la semilla sino la acumulación de materia seca.
\end{abstract}

Palabras claves: Zea mays L., vigor, germinación, madurez fisiológica, maíz.

\begin{abstract}
Changes in seed quality during seed development and maturation in maize. The objective of present study was determine the changes in seed developing and maturation on germination, seedling vigor, volumetric weight and weight of 1000 seeds. The experimental study was carried out at Celaya, Guanajuato, Mexico during 2001. The variety used in this study was sown at a rate of two seeds per hill in two replications of two rows of five meters long and spaced 0.75 meters. Sampling was started 30 days after female flower appearance then taking eight ears per treatment that were manually shelled. Average values for germination and seedling vigor showed the same behavior as indicatiors of seed ripening. Volumetric weight was increased to $71 \mathrm{~kg} / \mathrm{ha}$ at 130 days after planting. No significant effect was found on seed physiological quality due to 1000 seeds weight. The greatest seed germination percentage and seedling vigor was found when seed humidity was reduced from 40 to $20 \%$. Samples taken 106 and 121 days after sowing were poor indicators of seed quality. Also the greatest vigor and seed germination was found in the basal part of the ears. These results show that physiologic maturity is not the best indicator for maximum seed quality but dry matter accumulation.
\end{abstract}

Key words: Zea mays L., vigour, germination, fisiological maturity, maize.

\section{INTRODUCCIÓN}

La selección de plantas y mazorcas típicas de maíz para obtener la semilla para el próximo ciclo agrícola es la forma práctica y normal de los agricultores tradiciona- les en México, sin embargo, la selección masal que realizan permite solo conocer un progenitor lo que provoca en su mayoría rendimientos bajos. Entre las alternativas que se proponen para aumentar la producción y productividad del maíz, está el uso extensivo y generalizado de

\footnotetext{
1 Recibido para publicación el 16 de enero del 2004.

2 Instituto Tecnológico Agropecuario No. 33. Roque, Celaya, Guanajuato., México. Doctorado en Fitomejoramiento. Email: mmendoza66@ hotmail.com

3 Instituto Tecnológico Agropecuario de Conkal (SICA-ITA 2), México.

4 Facultad de Estudios Superiores Cuautitlán. UNAM. Apdo. Postal 25 Cuautitlán Izcalli, Edo. de México. México.

5 División Académica de Ciencias Biológicas (DACBIOL) Km 0.5 Carret. Villahermosa-Cárdenas, Tab.

6 Instituto Tecnológico Agropecuario No.23, C.P. 68000, Exhacienda de Nazareno, Xoxocotlan, Oaxaca.

7 Programa Suramericano de Maíz, Centro Internacional de Mejoramiento de Maíz y Trigo (CIMMYT), Apdo. Aéreo 5713, Cali, Colombia.
} 
semilla con alta calidad fisiológica por parte de los agricultores y la formación de variedades mejoradas con potencial productivo y de calidad (Molina et al. 1990). Por otra parte, la disponibilidad de una semilla con alta calidad es esencial para la utilidad de la industria semillera y el mantenimiento o crecimiento de una agricultura productiva (Barnes 1986). En este sentido existe poca información relacionada con el aspecto de calidad de semilla, para ello, son cuatro los componentes que originan que una semilla sea de máxima calidad, el genético, fisiológico, físico y fitopatológico, no obstante, las causas de muerte de la semilla son muy diversas, pero se ha estudiado más desde el punto de vista fisiológico y de sanidad.

La rápida emergencia uniforme y alto nivel de emergencia de la semilla son el reflejo del alto vigor de la misma, contrariamente las diferencias en el vigor de la semilla es la causa de la emergencia no uniforme en campo (Matthews 1980). Investigaciones en el desarrollo y maduración de la semilla revelan que existe una gran controversia en cuanto a cuando ocurre o se tiene la máxima calidad de la semilla, por un lado mencionan que esta ocurre cuando se presenta la etapa final del llenado de la semilla, es decir, en la etapa de madurez fisiológica en soya y trigo (Rasyad et al. 1990); otros autores como Ellis et al. (1993) en semilla de arroz y en el cultivo del frijol, Sanhewe y Ellis (1996) concluyen que la máxima calidad en estos cultivos se encuentra cuando existe la máxima acumulación de materia seca.

La calidad fisiológica de una semilla resulta de la historia de la planta madre, primero la adquisición de la habilidad para producir semillas vigorosas y tolerar el secado, entonces la pérdida de vigor es un proceso de envejecimiento que empieza durante el secado de la semilla (Powell et al. 1984), además, esta calidad de la semilla depende del clima y de las condiciones de crecimiento y desarrollo del cultivo (Delouche 1980). Las causas de las diferencias en el vigor de las semillas son definidas por la constitución genética, el ambiente, el contenido de humedad de la semilla y daño físico (Powell 1988 y Hampton 1991). Johnson y Tanner (1972) mencionan que la capa negra en maíz no indica la máxima acumulación de materia seca, por lo tanto, no se puede mencionar como regla que existe calidad de la semilla con este factor, por ejemplo, Carter y Poneleit (1973) reportan variabilidad en la presencia de la capa negra en líneas endogámicas de maíz, reportes similares realizan Knittle y Burris (1976) al evaluar híbridos a través de localidades en relación a la madurez del grano. Con base a lo anterior se planteó el presente trabajo con el objetivo de determinar cual es el efecto de la madurez fisiológica en la germinación, vigor, peso volumétrico y peso de 1.000 semillas.

\section{MATERIALES Y MÉTODOS}

\section{Localización del experimento}

El trabajo de investigación se realizó en dos etapas: la primera consistió en la conducción del experimento en el campo experimental de la Subdirección de Investigación y Graduados Agropecuarios, del ITA No. 33 de Roque, Celaya, Gto. México, ubicado a los $20^{\circ} 30^{\prime}$ 28 " de latitud Norte y $100^{\circ} 50^{\prime} 00^{\prime \prime}$ de longitud Oeste, a una altitud de $1750 \mathrm{~m}$. La segunda etapa del trabajo, se refiere a la determinación de variables asociadas con la calidad de la semilla, en el Laboratorio de Análisis de Semillas de la SIGA.

\section{Características climáticas}

De acuerdo a la clasificación de Köppen, modificada por García (1973), el clima de esta región es semicálido $\mathrm{BS}_{1} \mathrm{hW}(\mathrm{e})$, con una precipitación media anual de 550 a $710 \mathrm{~mm}$; las mayores precipitaciones ocurren de junio a septiembre y las más bajas de diciembre a abril. El régimen térmico medio anual es de $18,4^{\circ} \mathrm{C}$.

\section{Características edáficas}

Con base a la clasificación de los suelos de la FAOUNESCO (1970), modificada por la Dirección General de Geografía, en el municipio de Celaya, Gto., predominan suelos de tipo Vertisol Pélico, cuyas características son: suelos arcillosos de coloración oscura que presentan el fenómeno de contracción y expansión, por lo que se observan grietas profundas en algunas épocas del año, a menos que el suelo sea irrigado. Desarrollan un paisaje típico consistente en montículos y depresiones suaves, aunque también se encuentran inclinados. Además de estos suelos, se encuentran suelos de tipo Phacozen, en menor proporción. Los suelos son de origen volcánico, ricos en potasio, calcio y magnesio, con textura predominante arcillosa, gran capacidad de retención de humedad; un contenido pobre de materia orgánica, $\mathrm{pH}$ de 8,2 (medianamente alcalino), punto de marchitamiento permanente de $21 \%$, y densidad aparente de $1,1 \mathrm{~g} \mathrm{~cm}^{-3}$ (Ramírez 1985).

\section{Preparación del terreno}

Para tener una buena cama de siembra, se realizó un barbecho a una profundidad de $25 \mathrm{~cm}$, posteriormente se dieron dos pasos de rastra y nivelación. Se surcó a una distancia de $0,75 \mathrm{~m}$. 


\section{Siembra}

El trabajo se realizó con fecha de siembra en mayo del 2001. En la siembra se depositarán dos semillas cada $18 \mathrm{~cm}$, por lo que se tendrá una densidad de población de 74.075 planta/ha.

\section{Fertilización química}

Se realizó un análisis de suelo para conocer la fertilidad del terreno, ajustándolo a la fórmula 240-40-00 que es utilizada comercialmente para un desarrollo óptimo del cultivo.

\section{Control fitosanitario}

Se dio un tratamiento a la semilla de manera preventiva con Captán a la dosis recomendada por el fabricante y el control de plagas se realizó de acuerdo a la especie que se presentó.

\section{Material genético}

La semilla utilizada proviene de la variedad sintética de maíz Roque 1, la cual fue sembrada en el 2001, en el campo experimental del Instituto Tecnológico Agropecuario No. 33, ubicado en Roque, Celaya, Gto. Se sembró a una distancia entre surco de $0,75 \mathrm{~m}$ y a $0,18 \mathrm{~m}$ entre plantas, a una densidad aproximada a las 74.000 plantas/ha. Se iniciaron a los 30 días después de la floración (106 días después de la siembra), poco antes de la madurez fisiológica. Se cosechó ocho mazorcas al azar para cada muestreo con intervalos de seis días teniendo un total de cinco tratamientos. A cada muestra se le determinó el porcentaje de humedad al momento de la cosecha y se procedió a desgranar separando la semilla de la parte basal (SPB), de la parte media (SPM) y de la parte apical (SPA), resultando un total de 15 tratamientos. A cada tratamiento se le determinó el peso volumétrico, para ello se utilizó un determinador eléctrico modelo GMA-128. En ocho repeticiones se procedió a determinar el peso de 1.000 semillas. Para medir la germinación de la semilla previamente homogenizada a mano en una bolsa de papel, se tomaron tres repeticiones de 50 semillas; para ello se empleó el método descrito por Moreno (1996); para lo cual las 50 semillas de cada repetición se colocaron en toallas de papel humedecidas, las que se enrollaron y se colocaron por siete días a $25^{\circ} \mathrm{C}$ en una cámara de germinación, con una humedad relativa de 90-95\%. Para la evaluación de la germinación de plántulas normales, se realizaron dos conteos, el primero al quinto día y el segundo al séptimo día después de la siembra. Para la evaluación del vigor se utilizaron 25 semillas de cada repetición (tres repeticiones en total), mediante la medición de la longitud de plúmula, de acuerdo a Moreno (1996). En hojas de papel para germinación, se trazó una línea a la mitad e igualmente se marcan cinco líneas paralelas a la línea central hacia la parte superior de la hoja con un intervalo de $2 \mathrm{~cm}$ entre línea a línea. Sobre la línea central se colocó cinta masking de manera que la semilla se pueda pegar sobre ella. Cada repetición se colocó en línea, la central con el embrión hacia arriba y con la plúmula apuntando a la parte superior de la hoja donde están las líneas paralelas a la central. Las semillas se cubrieron con dos hojas de papel para germinación y se enrollan; los rollos se colocan en bolsas de polietileno y verticalmente en germinadoras con humedad relativa de $90-95 \%$, a $25^{\circ} \mathrm{C}$ por siete días. La evaluación sólo se hizo con plántulas normales. Para obtener el valor del vigor, el número de plúmula que queda entre las dos líneas paralelas se multiplica por el valor de dichas paralelas y los productos se suman. La longitud media de la plúmula (L) se obtiene dividiendo el producto de la suma entre el número de plántulas normales que resultaron (Moreno 1996); es decir, aquel tratamiento que presente un valor de nueve es el que tiene más vigor, como indica la siguiente fórmula:

$$
L=\left(n x_{1}+n x_{2}+\ldots n x_{11}\right) / 25
$$

\section{En donde:}

$L=$ longitud media de las plúmulas

$n=$ número de plúmulas entre cada par de paralelas

$x=$ la distancia media desde la línea central

El análisis estadístico que se aplicó en campo fue un bloques al azar con dos repeticiones, y en el laboratorio fue un diseño completamente al azar con arreglo factorial con tres repeticiones, con la finalidad de obtener información de las cosechas que fue el factor A, la posición de la semilla (PS) que fue el factor B y la interacción entre ambas fuentes de variación. Posteriormente se realizó la comparación de medias a través de la DMS.

\section{RESULTADOS Y DISCUSIÓN}

Tres características que definen la calidad física y fisiológica de la semilla fueron medidas en el laboratorio (peso de 1.000 semillas, germinación y vigor). El análisis de varianza para estas tres variables muestra diferencias altamente significativas para la fuente de variación muestreos, posición del grano en la mazorca y la interacción entre muestras y ubicación del grano. Entre los muestreos existió una diferencia en la maduración y 
desarrollo de la semilla con una respuesta diferente en cada tratamiento hacia el \% de germinación, el vigor y peso de 1.000 semillas. La diferencia observada en la posición de la semilla en la mazorca también mostró al menos un tratamiento diferente con respecto a las tres características antes mencionadas. La interacción de las etapas de maduración de la semilla con la semilla de la parte basal, media y apical de la mazorca también se observó estadísticamente una respuesta diferente. Esto significa que el grado de madurez de la semilla de maíz estuvo fuertemente afectado por el contenido de humedad al menos para estas características fisiológicas, de la misma forma hubo una respuesta de la posición de la semilla a estas variables (Cuadro1).

Cuadro 1. Promedio de los cuadrados medios del análisis de varianza para las variables en estudio en una variedad de maíz, en Celaya, Guanajuato, México. 2001.

\begin{tabular}{lrlll}
\hline \multicolumn{1}{c}{ F. V. } & G.L. Germinación & Vigor & $\begin{array}{c}\text { Peso de 1.000 } \\
\text { semillas }\end{array}$ \\
\hline Repeticiones & 2 & 0,27 & 0,55 & 1,29 \\
Muestreos (M) & 15 & $60,91^{* *}$ & $93,92^{* *}$ & $39,29 * *$ \\
PS & 2 & $2,99 * *$ & $7,34^{* *}$ & $2.565,74^{* *}$ \\
M*PS & 30 & $0,78^{* *}$ & $5,44^{* *}$ & $9,47 * *$ \\
Error & 94 & 0,39 & 0,97 & 1,48 \\
Total & 143 & & & \\
DMS & & 0,58 & 0,92 & 0,69 \\
\hline
\end{tabular}

*,**, Existió diferencia significativa y altamente significativa al 5 y 1 porciento, respectivamente. $\mathrm{M}=$ maduración y desarrollo de la semilla (muestreos); PS= posición del grano en la mazorca.

El primer muestreo (106 días después de la siembra o 30 días después de la floración) presentó el porcentaje más bajo de germinación $(7,02 \%)$ y el valor más bajo en vigor $(0,1)$ (Figura 1$)$. Por su parte, el porcentaje de germinación de la semilla presentó un crecimiento lineal a medida que la semilla concentraba una

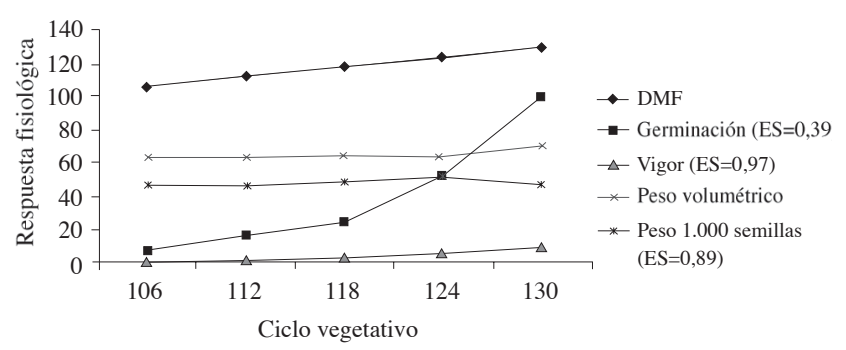

Figura 1. Distribución de las variables físicas y fisiológicas de la semilla de maíz en una variedad sintética en la localidad de Celaya, Guanajuato, México, ciclo 2001 mayor cantidad de solutos, esto significa, que a medida que la madurez fisiológica alcanza su máximo en la semilla, también se tuvo un aumento en el porcentaje de germinación; donde existió una diferencia de 24 días entre el primer muestreo y el último, y se encontró que la germinación ascendió de 7,02\% al $100 \%$, respectivamente. Un mismo patrón de comportamiento se observó para vigor, es decir, a medida que transcurrieron los días después de la siembra se revelaba un mayor índice del mismo. Entonces, estos resultados demuestran una asociación positiva entre el tiempo a la cosecha (ciclo vegetativo), la germinación y el vigor, lo cual indica que existe un equilibrio entre lo agronómico y la calidad fisiológica de la semilla; resultados similares reportan Delouche (1980) y Hampton (1991). El peso volumétrico fue similar para los primeros cuatro muestreos (64 kg/ha), en el último muestreo que se realizó a los 130 días después de la siembra se alcanzó el máximo peso superando a los demás tratamientos por 7 $\mathrm{kg} / \mathrm{ha}$; al respecto, Tekrony y Egli (1997) concluyen que la máxima calidad de la semilla de maíz se obtiene cuando existe el máximo peso seco. En el peso de 1.000 semillas la variación entre tratamiento osciló de 46,40 $\mathrm{g}$ a los 112 días a $51,97 \mathrm{~g}$ a los 124 días después de la siembra, es decir, esta característica no se afecto a medida que se realizaron los muestreos.

En la Figura 2 se presenta la interacción del porcentaje de humedad de la semilla a través del ciclo vegetativo, aquí se observa que el primer muestreo presenta $40 \%$ de humedad el cual fue realizado a los 30 días después de la floración (106 días después de la siembra), la humedad se redujo gradualmente conforme transcurrieron los muestreos ó el ciclo vegetativo, tal que el último tratamiento realizado a los 130 días después de la siembra se encontró un $20 \%$ de humedad en la semilla. Este resultado indica la importancia de la cantidad de agua en la semilla en el componente fisiológico. Se observa un crecimiento para la germinación y vigor a medida que este factor se reduce en la semilla y alcanza su máximo cuando existe la mayor acumulación de materia seca., es decir, el equilibrio entre la humedad de la semilla, la germinación y el vigor no ocurre en la etapa de madurez fisiológico como lo señalan Knittle y Burris (1976) quienes concluyen que la semilla de maíz puede ser cosechada antes y después de madurez sin afectarse el vigor. Algunos resultados que no concuerdan son los reportados por Knittle y Burris (1976) quienes indican que la semilla de maíz cosechada antes de la madurez fisiológica puede ser tan vigorosa como la cosechada al momento de madurez fisiológica o después de la misma. La madurez fisiológica de la semilla esta definida en algunos cultivos por el máximo peso seco, que es uno de los factores que influye en el vigor de la semilla (Kaliangile y Mulioleka 1995), no obstante Coolbear (1975), observa en cebada que la 


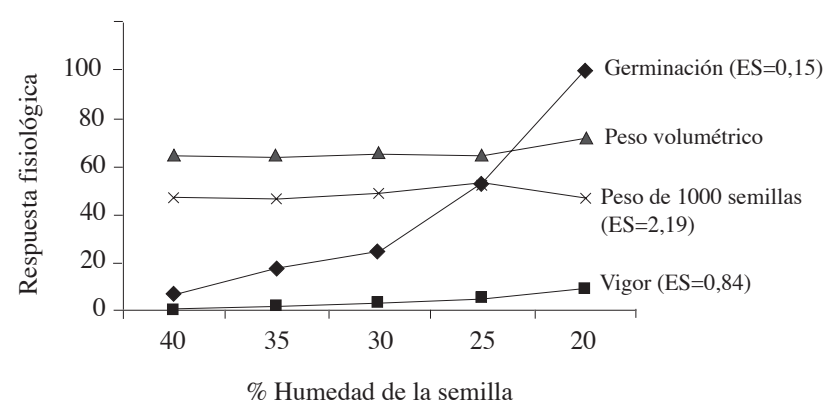

Figura 2. Efecto de la humedad de la semilla en las características físicas y fisiológicas en una variedad de maíz en Celaya, Guanajuato, México. 2001.

semilla continua con la acumulación de reservas específicas después de la madurez fisiológica y/o cuando la semilla ha alcanzado su máximo peso seco.

Por otra parte, el peso volumétrico y el peso de 1.000 semillas no influye tan drásticamente para obtener una semilla de alta calidad, como las variables antes señaladas, no obstante, el peso volumétrico presenta siete unidades de diferencia a través de los diferentes muestreos.

En la Figura 3 se muestra el comportamiento de la germinación, peso de 1.000 semillas y vigor a través de los cinco muestreos considerando la ubicación de grano en la mazorca. Para las tres variables se encontró diferencia estadística, esto significa que existió una respuesta diferencial a estas variables de la semilla de la base, la ubicada en la parte media y la posición apical. Aunque estos resultados se ven afectados por el contenido de la humedad de la semilla, se observa que el porcentaje de germinación de la semilla de la base y media de la mazorca presentaron un comportamiento estadísticamente similar $(63,8$ y 60,9 , respectivamente), no así, la semilla ubicada en la parte apical (56,2\%), el valor DMS fue de 0,252. Para el peso de 1.000 semillas,

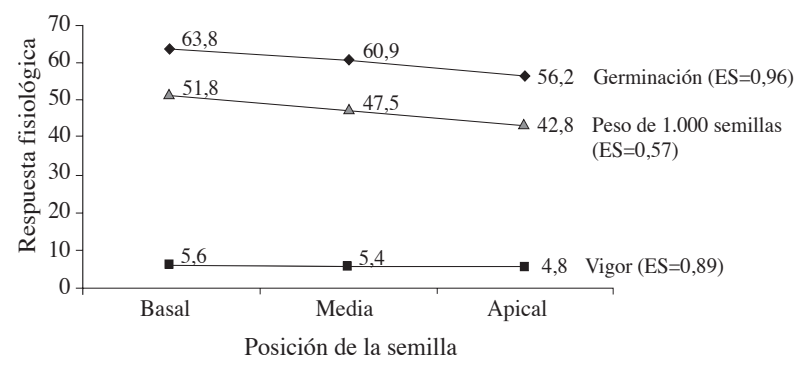

Figura 3. Respuesta de la germinación, peso de 1000 semillas y vigor considerando la posición de la semilla en la mazorca a través de muestreos en maíz en el ambiente de Celaya, Guanajuato, México, durante el ciclo otoño-invierno. 2001. también existe un comportamiento estadístico diferencial, aquí se observan tres grupos, el primero semilla de la base $(51,82)$, segundo semilla media $(47,56)$ y tercero semilla apical $(42,87)$ con un valor de 0,29 de DMS. Respecto al vigor se encontraron dos grupos estadísticamente diferentes, existiendo un patrón de comportamiento similar al de germinación. El máximo vigor se alcanzó en la semilla de la base y media de la mazorca (5,65 y 5,40, respectivamente), en el otro grupo se detectó la semilla de la parte apical con un valor de 4,88, para esta variable se estimó un valor DMS de 3,99. Por su parte Moreno et al. (1998) demuestran que la semilla de maíz plana y grande presenta una mayor longevidad que la semilla redonda y chica. Hay evidencia en soya que señala que las semillas de menor tamaño tienen mejores características para su longevidad, lo anterior se observó en México cuando se almacenaba la variedad Tropicana, que era de grano pequeño. Esta misma característica ha sido observada con otras variedades de soya (Singh 1976). Estos resultados indican que un programa de producción de semilla no debe separar la semilla de la base y parte media de la mazorca, pero si la semilla de la parte apical, éstas tienen una relación estadística con la germinación y el vigor, siendo inferior la respuesta de la semilla ubicada en la parte apical de la mazorca. De ser utilizada la semilla de la parte apical para la siembra se debe realizar la recomendación de utilizar una mayor cantidad de semilla en la siembra con la finalidad de lograr un buen establecimiento de cultivo, ya que de alguna manera el tener una buena uniformidad en campo asegura excelentes rendimientos y esto se logra con un máximo en el vigor (Mattews 1980).

\section{CONCLUSIONES}

Se encontró un mismo patrón de comportamiento para el porcentaje de germinación y longitud de plúmula (vigor) a medida que se alcanzó el máximo peso seco de la semilla; es decir, el 100\% de germinación y el máximo en vigor (9) se logró cuando el porcentaje de humedad de la semilla fue del $20 \%$ (quinto muestreo). Este factor afecta fuertemente a estas variables, no tanto al peso de 1.000 semillas y peso volumétrico. Entonces el equilibrio entre el contenido de agua de la semilla y el componente fisiológico se alcanza cuando la semilla contiene el $20 \%$ de humedad donde han transcurrido 130 días después de la siembra; respuesta similar se obtuvo con la ubicación de la semilla en la mazorca, siendo mejor la parte basal y parte media. El máximo en germinación y vigor no se alcanza con la madurez fisiológica de la semilla, si no esto se atribuye a la máxima acumulación de materia seca. 


\section{LITERATURA CITADA}

BARNES, R. F. 1986. Foreword: In: M.B. McDonald, Jr.; C.J. Nelson (eds.). Physiology of seed deterioration. CSSA special publication, USA. No. 11. p. 7.

CARTER, M. W.; PONELEIT, C. G. 1973. Black layer maturity and filling period and variation among inbred lines of corn (Zea mays L.). Crop Science 13: 436-439.

COOLBEAR, P. 1995. Mechanisms of seed deterioration. In: Seed quality: basic mechanisms and agricultural implications, (ed A.S. Basra). The haworth Press Inc. pp. 2.223-2.227.

DELOUCHE, J. C. 1980. Environmental effect on seed development and seed quality. Hortscience 15: 775-780.

ELLIS, R. H.; SINNIAH, U. R.; JOHN, P. 1993. Irrigation and seed quality development in rapid-cycling Brassica. In: M. Black, K.J. Bradford, J.Vazquez-Ramos (eds). Seed biology: Advances and applications. pp.113-121.

FAO-UNESCO. 1970. Clasificación de los suelos. Organización de las Naciones Unidas. México. 280 p.

GARCÍA, E. 1973. Modificaciones al sistema de clasificación climática de Köppen. Ed. UNAM. Instituto de Geografía. México, D. F. 245 p.

HAMPTON, J. G. 1991. Herbage seed lot vigour - do problems start with seed production? Journal of Applied Seed Production 9:87-93.

JOHNSON, D. R.; TANNER, J.W.1972. Calculation of the rate and duration of grain filling in corn (Zea mays L.). Crop Science 12:185-186.

KALIANGILE, T.; MULIOLEKA, S. W. 1995. Seed development. In: S.W. Mulioleka (ed). Zambia seed technology Handbook. Ministry of Agriculture, Food and Fisheries, Zambia. pp. 28-34.

KNITTLE, K. H.; BURRIS, J. S. 1976. Effect of kernel maturation on subsequent seedling vigor in maize. Crop Science 16:851-885.
MATTHEWS, S. 1980. Controlled deterioration; a new vigour test for crops seeds. In: P. D. Hebblethwaite (eds). Seed Production. London; Butterworths. pp. 467-660.

MOLINA, M. J.; ESTRADA, J. A.; LIVERA, M. M.; GONZÁLEZ, V. A. 1990. Análisis de la enseñanza, producción e investigación de semillas en México. SOMEFI. Chapingo, México. 205 p.

MORENO, M. E. 1996. Análisis físico y biológico de semillas agrícolas. Ed. UNAM-FAO. México, D.F. 393 p.

MORENO, M. E.; VÁZQUEZ, B. M.; RIVERA, R. A.; NAVARRETE, M. R.; ESQUIVEL, V F. 1998. Effect of seed shape and size on germination of corn (Zea mays L.) stored under adverse conditions. Seed Science and Technology 26: 439-448.

POWELL, A. A.; MATTHEW, S.; OLIVEIRA, M.A. 1984. Seed quality in grain legumes. Advances in Applied Biology 10:217-285.

POWELL, A. A. 1988. Seed vigour and field establishment. Advances in research and technology of seeds 16:419426.

RAMÍREZ, S. 1985. Evaluación del efecto de tres distancias entre surco en la producción de sorgo (Sorgum bicolor M). Tesis profesional. I.S.E.T.A., Roque, Celaya, Gto. pp. 122.

RASYAD, A.; VAN SANFORD, D. A.; TEKRONY, D. M 1990. Changes in seed viability and vigour during wheat seed maturation. Seed science and technology 18:259-267.

SANHEWE, A. J.; ELLIS, R. H. 1996. Seed development and maturation in Phaseolus vulgaris II Post-harvest longevity in air dry storage. Journal of Experimental Botany 47:959-965.

SINGH, B.B. 1976. Breeding soybean varieties for the tropics. INTSOY Ser. 10: 11-17.

TEKRONY, D. M.; EGLI, D. B. 1997. Accumulation of seed vigour during development and maturation: In: R. H. Ellis, M. Black, A. J. Murdoch, T. D. Hong (eds). Basic and applied aspects of seed biology. Kluwer Academic Publishers, Boston. pp: 369-384. 